\section{A rare hereditary disease: Muckle-Wells syndrome}

\author{
Nitya Ramreddy, ${ }^{1}$ Aviva Hopkins, ${ }^{2,3}$ \\ Carlos Lozada² \\ 'Department of Internal Medicine; \\ 2Department of Rheumatology, \\ University of Miami Miller School of \\ Medicine, Miami; ${ }^{3}$ Holy Cross Hospital, \\ Fort Lauderdale, FL, USA
}

\section{Abstract}

Cryopyrin associated periodic syndrome (CAPS) is a dominantly-inherited autoinflammatory disease, which is included in the group of periodic fever syndromes. It is caused by a defect in the regulation of inflammatory cytokines, particularly interleukin-1 $\beta$. CAPS encompasses a spectrum of three phenotypes of increasing severity: familial cold autoinflammatory syndrome, Muckle-Wells syndrome (MWS) and neonatal onset multisystem inflammatory disease. We report the case of a 58-year-old male, who had migratory joint pains, daily urticaria, chills, and episodic conjunctivitis since childhood and hearing loss in his 20 s with a family history of similar symptoms. He was diagnosed with MWS after being found to have a $R 262 W$ gene mutation in NLRP3 gene and successfully treated with canakinumab. After his discovery, other $1^{\text {st }}$ and $2^{\text {nd }}$ degree family members with similar complaints were found to have the same genetic mutation and were also successfully treated with canakinumab.

\section{Introduction}

Cryopyrin associated periodic syndromes (CAPS) are rare, dominantly-inherited autoinflammatory diseases characterized by recurrent episodes of systemic inflammation involving multiple tissues including joints, skin, the central nervous system, eyes, and ears. Unlike autoimmune diseases, CAPS are related to dysfunction of the innate immune system. The syndromes are due to dominant mutations in NLRP3, which encodes a key component of the innate immunity that regulates the secretion of interleukin-1 (IL-1 $\beta$ ). There is a spectrum of three phenotypes, sometimes clinically overlapping, of increasing severity: familial cold autoinflammatory syndrome (FCAS), MuckleWells syndrome (MWS) and neonatal onset multisystem inflammatory disease (NOMID). MWS has an intermediate phenotype of severity characterized by intermittent episodes of fever, urticarial rash, joint pain, progressive hearing loss, and, sometimes, secondary amyloidosis with nephropathy.

\section{Case Report}

A 58-year-old male with past medical history of hearing loss at age of 23 while serving in the military working with aircraft, presented to the office complaining of fatigue and right wrist pain and swelling. He reported years of migratory joint pains and swelling, the first episode of which occurred at age 19. He had since experienced different joint pains with and without accompanying joint swelling on an almost weekly basis. Review of systems was notable for fatigue, frequent headaches and a daily rash. The patient described daily episodes of non-pruritic, painful, erythematous spots all over his body since childhood. Rash was worse in the evenings, associated with chills, and not aggravated by exposure to cold. After being noted to have conjunctival injection, with further questioning, patient reported that he had experienced intermittent eye redness since his time in the military, which he attributed to a presumed chemical exposure. He denied fevers, myalgias, chest pain or shortness of breath. No history of recent vaccinations or travel. He endorsed drinking alcohol occasionally but never smoked cigarettes or used any illicit drugs. Family history was notable for mother and sister with similar complaint of daily rash (Figure 1). The patient was afebrile and hemodynamically stable on presentation. His right eye was mildly injected, pupils were equal and reactive to light, accommodation and no photophobia. He had bilateral hearing aids. He did not have swelling of any joints on presentation and range of motion was intact. He had erythematous papules scattered all over his trunk and lower extremities (Figure 2). Initial blood work showed leukocytosis with white cell count of 19,300/uL (normal range 3800-10,800/uL) with $80.9 \%$ neutrophils, C- reactive protein of 3.76 $\mathrm{mg} / \mathrm{dL}$ (normal $<0.80 \mathrm{mg} / \mathrm{dL}$ ), erythrocyte sedimentation rate of $45 \mathrm{~mm} / \mathrm{h}$ (normal $<20$ $\mathrm{mm} / \mathrm{h}$ ), normal serum chemistry, liver function tests, non-reactive hepatitis panel and negative Quantiferon Gold testing. Considering his family history of mother and sister with daily rash, his hearing loss, urticarial rash and conjunctival injection on exam, his reported frequent headaches, and noted leukocytosis, CAPS was suspected and genetic testing for $N L R P 3$ was ordered. He was found to be heterozygous for $R 262 W$ mutation in the NLRP3 gene, confirming the diagnosis of MWS. Patient was started on an anti-IL-1b monoclonal antibody Canakinumab with complete resolution of fatigue, joint pain, conjunctivitis, and
Correspondence: Nitya Ramreddy, Department of Internal Medicine, University of Miami Miller School of Medicine/Holy Cross Hospital, 4725 N Federal Hwy, Fort Lauderdale, FL 33308, USA.

Tel.: + 1.312.613.5131.

E-mail:nrr49@med.miami.edu

Key words: Cryopyrin associated periodic syndrome; Muckle-Wells syndrome; NLRP3 gene; Canakinumab.

Contributions: the authors contributed equally

Conflict of interest: the authors declare no potential conflict of interest. Carlos Lozada has participated in AbbVie osteoarthritis related clinical research and has consulted with Pfizer on Rheumatoid arthritis related issues.

Received for publication: 16 April 2016.

Revision received: 25 April 2016.

Accepted for publication: 25 April 2016.

This work is licensed under a Creative Commons Attribution NonCommercial 4.0 License (CC BYNC 4.0).

(C) Copyright N. Ramreddy et al., 2016

Licensee PAGEPress, Italy

Rheumatology Reports 2016; 8:6535

doi:10.4081/rr.2016.6535

urticarial rash within days following his initial injection. Leukocytosis improved and inflammatory markers normalized. The patient's brother and niece who were discovered with be similarly afflicted learned of his remarkable response through social media. NLRP3 gene mutation was then detected and they were subsequently started on Canakinumab for their symptoms, with similar remarkable response. The patient's sister who has experienced hives since infancy has been unable to afford treatment because of insurance issues.

\section{Discussion and Conclusions}

CAPS are a group of rare hereditary disorders that includes three phenotypes: FCAS, MWS and NOMID. ${ }^{1}$ Drs. Muckle and Wells first described an autosomal dominant syndrome in 1962 manifested as urticaria, chills, sensorineural hearing loss, and renal amyloidosis, which came to be known as MWS. ${ }^{2}$ CAPS occur due to a missense mutation in the NLRP3 gene located on chromosome 1q44, which codes for cryopyrin. ${ }^{3,4}$ Cryopyrin helps inflammasomes cleave IL-1 $\beta$. The defect in cryopyrin leads to elevation of interleukins with resultant inflammatory symptoms such as urticaria and arthralgia. ${ }^{5,6}$ Diagnosis is usually made by skin 
biopsy which shows perivascular and neutrophilic dermal infiltration ${ }^{7}$ and/or genetic testing in an individual with suggestive symptomatology. Genetic testing will often demonstrate a mutation in NLRP3 gene, mostly in exon 3., ${ }^{8,9}$ IL-1 inhibitors have been shown to be effective in the treatment of CAPS and include

Anakinra, Rilonacept and Canakinumab. ${ }^{10}$ Anakinra is an IL-1 antagonist, which binds to IL-1 receptor and blocks its activity. ${ }^{11}$ It is given as a daily subcutaneous injection and leads to symptom remission. Rilonacept also binds to IL-1 type 1 receptor and is given as a weekly injection. ${ }^{12}$ Canakinumab is a human IgGl anti
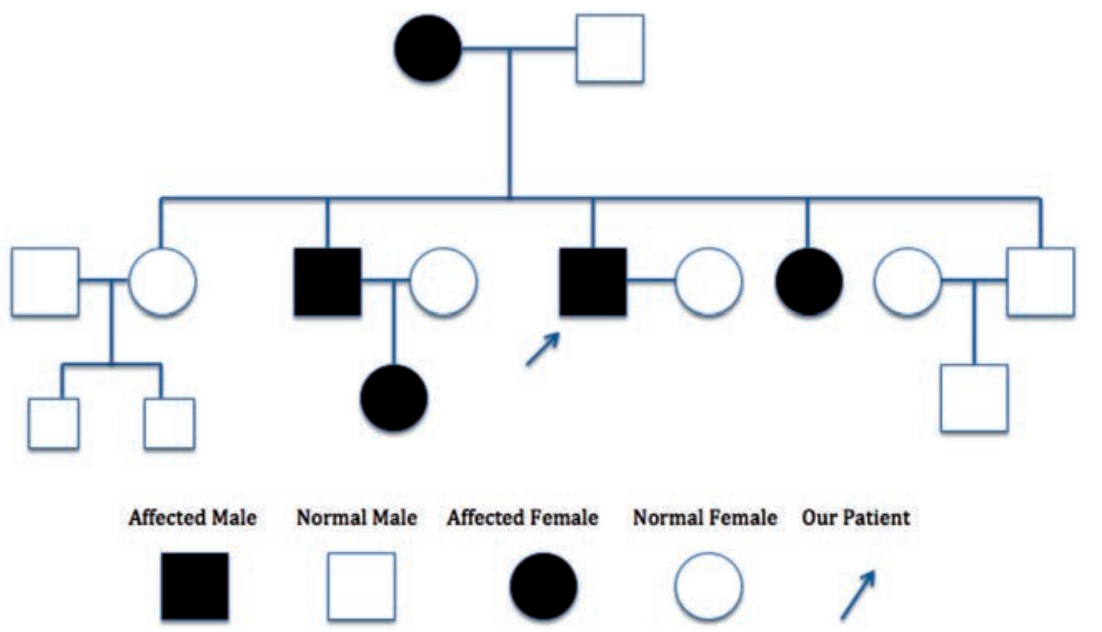

Figure 1. Family history and inheritance of Muckle-Wells syndrome amongst family members.

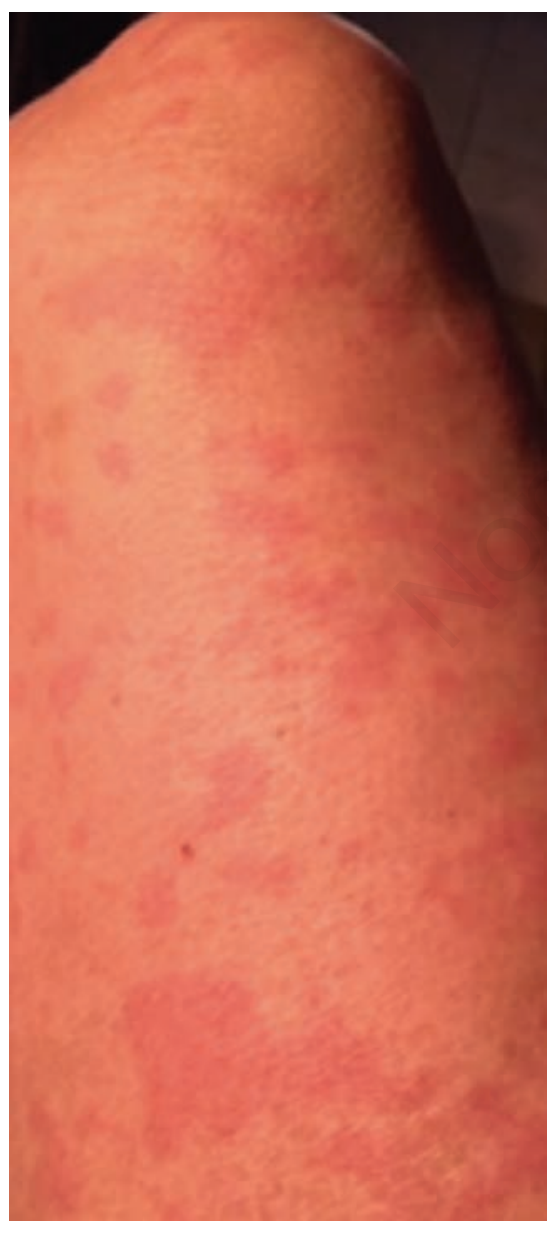

Figure 2. Erythematous papules on lower extremity.
IL-1b monoclonal antibody, with a half-life of 26 days. It is generally administered as a subcutaneous injection every 8 weeks, 11,12 but the frequency of canakinumab dosing can be dependent on the phenotype and severity of the disease. ${ }^{13}$ A multi center study conducted to determine the efficacy of Canakinumab in patients with CAPS showed that the real life effectiveness is lower than expected and treat to target strategy improves outcomes in patients. ${ }^{14}$ Our patient achieved complete resolution of symptoms within 2 days following his first dose of canakinumab. After he had continued remission following his second dose 8 weeks later, he decided to see how long he could go without symptom recurrence. He elected to receive his third dose 6 months later when fatigue began to return, leukocytosis worsened, and inflammatory markers increased. Following that injection, he opted for his next dose at 4 months because of again slight recurrence of fatigue. Currently, he has not had a headache, joint pain, eye redness or urticarial rash since the first injection. CAPS are rare hereditary diseases and the diagnosis is often delayed. However once the diagnosis is confirmed, there are very effective, lifealtering treatments.

\section{References}

1. Neven B, Prieur AM, Quartier dit Maire P. Cryopyrinopathies: update on pathogene- sis and treatment. Nat Clin Pract Rheumatol 2008;4:481-9.

2. Muckle TJ, Wells M. Urticaria, deafness, and amyloidosis: A new heredo-familial syndrome. Q J Med 1962;31:235-48.

3. Abdulla MC, Alungal J, Hawkins PN, Mohammed S. Muckle-Wells syndrome in an Indian family associated with NLRP3 mutation. J Postgrad Med 2015;61:120-2.

4. McDermott MF, Aganna E, Hitman GA, et al. An autosomal dominant periodic fever associated with AA amyloidosis in a north Indian family maps to distal chromosome 1q. Arthritis Rheum 2000;43:2034-40.

5. Agostini L, Martinon F, Burns K, et al. NALP3 forms an IL-1beta-processing inflammasome with increased activity in Muckle-Wells autoinflammatory disorder. Immunity 2004;20:319-25.

6. Mariathasan S, Newton K, Monack DM, et al. Differential activation of the inflammasome by caspase-1 adaptors ASC and Ipaf. Nature 2004;430:213-8.

7. Shinkai K, McCalmont TH, Leslie KS. Cryopyrin-associated periodic syndromes and autoinflammation. Clin Exp Dermatol 2008;33:1-9.

8. Church LD, Savic S, McDermott MF. Long term management of patients with cryopyrin-associated periodic syndromes (CAPS): focus on rilonacept (IL-1 Trap). Biologics 2008;2:733-42.

9. Aksentijevich I, Nowak M, Mallah M, et al. De novo CIAS1 mutations, cytokine activation, and evidence for genetic heterogeneity in patients with neonatal-onset multisystem inflammatory disease (NOMID): A new member of the expanding family of pyrin-associated autoinflammatory diseases. Arthritis Rheum 2002;46:3340-8.

10. Jesus AA, Goldbach-Mansky R. IL-1 Blockade in autoinflammatory syndromes. Annu Rev Med 2014;65:223-44.

11. Kuemmerle-Deschner JB, Wittkowsk H, Tyrrell PN, et al. Treatment of MuckleWells syndrome: analysis of two IL-1-blocking regimens. Arthritis Res Ther 2013;15:R64.

12. Yu JR, Leslie KS. cryopyrin-associated periodic syndrome: an update on diagnosis and treatment response. Curr Allergy Asthma Rep 2011;11:12-20.

13. Caorsi R, Lepore L, Zulian F, et al. The schedule of administration of canakinumab in cryopyrin associated periodic syndrome is driven by the phenotype severity rather than the age. Arthritis Res Ther 2013;15:R33.

14. Kuemmerle-Deschner JB, Hofer F, Endres $\mathrm{T}$, et al. Real-life effectiveness of canakinumab in cryopyrin-associated periodic syndrome. Rheumatology 2016;55: 689-96. 